\title{
Image quality with current adaptive optics instruments ${ }^{\star}$
}

\author{
E. Tessier \\ Royal Greenwich Observatory, Madingley road, Cambridge CB3 0EZ, UK \\ e-mail address: tessier@ast.cam.ac.uk
}

Received September 12, 1996; accepted February 12, 1997

\begin{abstract}
From natural guide star adaptive optics data, we study the properties of the on-axis point spread function and discuss the performance of adaptive optics images. A close relation between the full width half maximum and the Strehl ratio is shown. The ultimate exploitation of adaptive optics images requires the deconvolution and therefore the calibration of the point spread function. This calibration is commonly made by sequentially observing a point source close to the astronomical target. In the partial correction regime, the calibration mismatch is the main source of noise or bias in the deconvolution process and it is mainly induced by the varying seeing conditions. Complementary techniques like near-sighted deconvolution, speckle and rebinning are briefly reviewed.
\end{abstract}

Key words: atmospheric effects — instrumentation: miscellaneous - methods: data analysis; observational techniques: interferometric; image processing

\section{Introduction}

The turbulence in the atmosphere perturbs the incoming light wavefront from an astronomical source and blurs the images for ground-based telescopes whose resolution is therefore limited by the seeing angle (typically one arcsecond). The Hubble space telescope (HST) or any space projects are an efficient but still expensive way to get rid of the turbulence problem. However, alternative techniques have been developed to restore the original resolution of ground based telescopes. Adaptive optics (AO) is one of them and probably the most promising one. Operational AO systems have already produced astronomical results (Léna 1994; Roddier et al. 1995). An adaptive optics system analyzes the disturbed wavefront and applies in real time corresponding commands to a deformable mirror in order to cancel the phase perturbation (see e.g. Roddier 1994 for a description). Depending on the quality of the correction achieved, a partially or fully corrected image is

\footnotetext{
* Based on observations made at ESO and SOR.
}

obtained. The so-called guide source corresponds to the source used by the wavefront sensor (WFS) to analyse the perturbed wavefront. At the moment, most of current adaptive optics experiments or projects only provide the natural guide star (NGS) mode. The phase perturbation depends on the viewing direction. So, any distance between the guide source and the science object will add an anisoplanatic error in the AO correction. From this point of view, the best choice for the guide source is the science object itself.

One can find in the literature (Ellerbroek 1994; Wilson \& Jenkins 1996 for recent examples) calculations of the mean correction achieved by an AO system running in some given turbulence conditions. The atmospheric coherence time $t_{0}$ and the atmospheric coherence length $r_{0}$ are two parameters used to describe these conditions. Both are wavelength-dependent $(\lambda)$ and function of the airmass. As the turbulence effects get worst or one goes to shorter wavelengths (faster turbulence and/or smaller $r_{0}$ ), the correction is poorer. Adaptive optics works in many cases in the partial correction regime.

If we ignore anisoplanatism, the AO image of a science object is the science object intensity distribution convolved with the point spread function. The point spread function (psf) is defined as the AO image obtained with a point source and then corresponds to the instrumental response function. So, the psf directly defines the quality of the image. In the full correction case, the psf correspond to the theoretical diffraction limit which is the Airy pattern for a clear circular aperture telescope. In the partial correction case (the general one), the psf is degraded by the power of the uncorrected or non fully corrected terms. Qualitatively, a superimposed halo (the so-called seeing halo) and a widening of the psf are observed (Rigaut et al. 1991).

Some scalar parameters have been generally used to describe the long or short exposure psfs. The Strehl ratio $(\mathrm{Sr})$ is used to quantify the degree of correction achieved. Appendix A shows how to extract this parameter from point source AO images. A Strehl ratio equal to one means a full correction. In case of no correction, 
the Strehl ratio of the seeing limited image is about $\left(\frac{r_{0}}{D}\right)^{2}$ where $D$ is the telescope aperture diameter. The full width half maximum (fwhm) of the psf is an indication of the image sharpness and tells about the spatial resolution achieved. For two-dimensional psfs, this parameter is actually the fwhm of the azimuthally averaged profile. The fwhm of the diffraction-limited psf is $\approx \lambda / D$ while that of the seeing-limited psf (the seeing angle) is $\approx \lambda / r_{0}$. A psf could have a sharp core but widely spread out. The $50 \%$ energy radius ( $r 50)$ tells within which radius is concentrated $50 \%$ of the energy. As for the fwhm, this parameter is computed on the azimuthally averaged profile.

It is quite difficult to predict from simulation and/or analytic calculation all the AO servo system and turbulence statistics induced effects. The different wavefront realizations and other noise sources make the AO system correction a statistical process. The long-exposure psf is the instantaneous psf averaged over a time long enough to have a good signal to noise ratio. In the partial correction regime, the turbulence statistics are here considered as the predominant process, the integration time should be much larger than $t_{0}$. Typically, this means one minute and 10 seconds in the infrared and in the visible respectively. Equivalently, it is the time to smooth the speckle pattern produced by the non-coherent energy present in a partially corrected image which is responsible for the seeing halo. By contrast, the central part of the psf gets its typical shape in a few coherence times. Unfortunately, the atmospheric turbulence is often a non-stationary process even over for such short times and is also function of the airmass. These points will be illustrated in this paper.

This work is found on natural guide star AO data taken with two operational A0 instruments based on a ShackHartmann wavefront and a piezo-actuated deformable mirror. This paper outlines adaptive optics performance and the limitations induced by the atmosphere to the AO system in the partial correction regime. The anisoplanatism issue and other sources of limitations due to the photon, background or detector noises are not considered here. Most previous works have concentrated on comparison between prediction and observed AO correction in term of Strehl ratio. However, the shape of the point spread function and its stability for various correction regime are important for an astronomical use. Practical problems like psf calibration, deconvolution of AO image or post-processing, which are complex issues (Tessier 1995), are therefore adressed.

Instruments used to produce the data are described in Sect. 2. In Sect. 3, the temporal and geometrical characteristics of the point spread function are shown for various correction regime. Especially, the stability of the psf against the changing turbulence conditions is tackled, some relationships between psf scalars are shown. Image quality degrades dramatically below a certain Strehl ratio value. Some comparison with theoretical predictions is done. Post-processing techniques like image deconvo- lution require some specific observational and calibration procedures which are presented in Sect. 4. Overall performances of AO data as a function of the correction are given in Sect. 5. In Sect. 6, we see that calibration and blind deconvolution techniques can be mixed to try and get an improved near-sighted deconvolution process. Section 7 shows how to combine speckle techniques with AO to improve the performance in the low correction regime. Conclusions are drawn in Sect. 8, from these processed NGS AO data, AO are shown to push down the limits compare to the seeing-limited imaging or the speckle imaging.

\section{Instruments and data}

Data presented here have been carried out with the adaptive optics system from the European Southern Observatory (ESO) called at this time Come-on+ (COP) and the adaptive optics system from the Starfire Optical Range (SOR) called Generation II (Gen II). Both systems are based on a Shack-Hartmann wavefront and a piezoactuated deformable mirror.

The Come-on+ instrument (Beuzit et al. 1994 for a technical description) recently renamed ADONIS is currently used at the focus of the 3.6 meter ESO telescope in Chile for NGS AO imaging in the near-infrared. For simplicity, I have selected observations of bright binary sources. NGS AO data of sub-arcsecond binaries carried out on November 1993 and January 1994 with COP have been provided by C. Perrier and J. Bouvier (Observatory of Grenoble, France) respectively.

The Gen II instrument (Fugate et al. 1994) operates at the Starfire Optical Range 1.5 meter telescope facility located near Albuquerque, New-Mexico, U.S.A. and delivers AO corrected images in the $I$-band $(0.88 \mu \mathrm{m})$. Gen II provides a laser and natural guide star modes. B. Ellerbroek and J. Christou (U.S. Air Force Phillips Laboratory, NM, U.S.A.) provided a set of NGS Gen II observations carried out on December 1994 and July 1995.

Table 1. AO systems characteristics

\begin{tabular}{|l|l|l|}
\hline AO system & COP/Adonis & SOR Gen II \\
\hline WFS geometry (subapertures) & $7 \times 7$ & $16 \times 16$ \\
Actuators number & 52 & 241 \\
WFS maximum frame rate & $200 \mathrm{~Hz}$ & $1667 \mathrm{~Hz}$ \\
$-3 d B$ close loop bandwidth & $50-60 \mathrm{~Hz}$ & $80-120 \mathrm{~Hz}$ \\
\hline
\end{tabular}

Table 1 gives the main characteristics for the two AO systems. We note that the WFS analyses in the $V-R$ light. Here after, AO imaging data in the $J H K$ bands and the $I$ band come from COP and Gen II respectively. The experimental data correspond to various turbulence conditions $\left(r_{0}, t_{0}\right) . d / r_{0, \lambda}$ is currently 1 to 2 where $\mathrm{d}$ is the subaperture width and $r_{0, \lambda}$ is the seeing parameter at the imaging wavelength $\lambda$, and the Greenwood frequency 
$f_{\mathrm{g}}$ (which is inversely proportional to $t_{0}$ ) is often smaller than the AO system bandwidth. Unfortunately, for COP data, these atmospheric parameters are only estimations given by the observers. The guide source is always the astronomical target so that only the on-axis psf is considered here. Sources are bright so that continuous short exposure time frames were often used, there was no photon starvation in the WFS and photon, detector and background noises are expected to be small in order to stress the others fundamental limitations due to the turbulence or the AO control system.

\section{Psf characteristics}

Adaptive optics works in many cases in the partial correction regime, i.e. with a Strehl ratio smaller than 0.8. Here, I emphasize the characteristics of such partially corrected psfs. The temporal and geometrical properties are therefore described.

In the partial correction regime, seeing evolution during the night induces some variations in the mean correction (Rigaut et al. 1991). It is shown by following up the Strehl ratio which is an excellent tracer of the correction. We now try to look at what is going on during a typical integration time of a few minutes. Table 2 gives the mean and the standard deviation of the scalars for 1 s psfs recorded continuously for 5 minutes. One can see that for good $\mathrm{Sr}$, the fwhm is stable but not the $r 50$. By contrast, for lower Sr, the $r 50$ gets more stable and the fwhm fluctuates a lot.

Table 2. Mean and standard deviation normalized to the mean for the 1s psfs scalars in 5 minutes time are given for three correction degrees. Come-on + data

\begin{tabular}{|l|l|l|l|}
\hline Correction in Sr & 0.25 & 0.10 & 0.015 \\
\hline$r 50(\lambda / D)$ & 2.5 & 4.5 & 7.8 \\
fwhm $(\lambda / D)$ & 1.25 & 1.8 & 5.0 \\
$\sigma(\mathrm{Sr}) / \mathrm{Sr}$ & $15 \%$ & $26 \%$ & $22 \%$ \\
$\sigma(r 50) / r 50$ & $15 \%$ & $9 \%$ & $6 \%$ \\
$\sigma($ fwhm $) /$ fwhm & $6 \%$ & $16 \%$ & $24 \%$ \\
\hline
\end{tabular}

As seen in Fig. 1, the Sr of continuous short exposure $(2 \mathrm{~s})$ frames appears to be highly variable in time. First, these variations may be explained from the statistics of the wavefront error reconstruction. Literature lacks explicit calculation of the variance of the AO correction (Strehl ratio) for a given integration time and wavefront statistics (see however Johnston et al. 1996 for a recent example). But this is probably not responsible for such high variations even if some temporary close loop failures due to the finite bandwidth of the system may account for some. Seeing evolution is probably responsible for structures like slopes and breaks as seeing is known to vary in such a way. Any conclusion should be taken cautiously but the psfs stability may be more limited by the seeing statistics than by the AO system itself (Tessier 95). Actually, to decide this issue, it will be interesting to correlate Sr measurements to the $r_{0}$ and $t_{0}$ measurements from a seeing monitor looking at the same viewing direction.

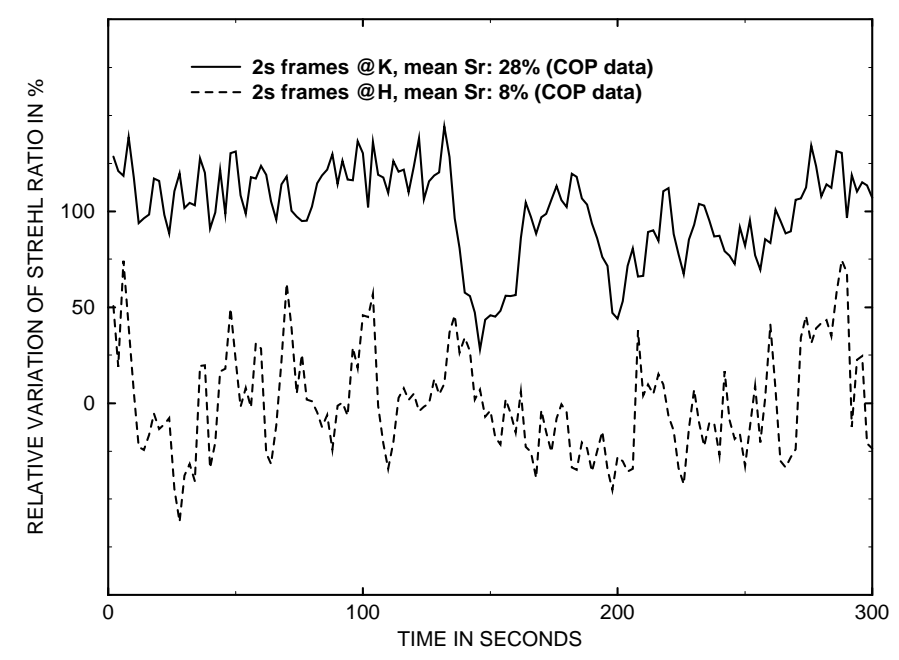

Fig. 1. Strehl ratio as a function of time from continuous 2 seconds exposure frames. Sr is normalized to its mean value to highlight relative variations. $\mathrm{Sr}$ is shifted for $H$ data for readability. Data at $H$ and $K$ are not simultaneous but were taken a few minutes apart. One uses the same scale for convenience. Come-on + data

From Fig. 2, the fwhms of short exposure psfs are quite well stabilized in $K$ when $\mathrm{Sr}$ is higher than $20 \%$; fwhms being always smaller than 0.18 arcsec (except for a few seconds). But in $H$, the Sr has dropped about $10 \%$ and some large variations of the fwhm between 0.13 arcsec and 0.25 arcsec are observed. This will be explained by the close relationship between these two parameters (further in this section).

Sets of Psfs with equivalent integration time ranging from $2 \mathrm{~s}$ to $30 \mathrm{~s}$ have been computed from a cube of $2 \mathrm{~s}$ frames taken continuously for 5 minutes in the $K$ band with a mean $\mathrm{Sr}$ of 0.25 and in the $H$ band with a mean $\mathrm{Sr}$ of 0.08 . The statistic $p(t, r)$ is defined to be the pixel value at a given radial distance $r$ to the central peak for a psf integrated over a time $t$. The minimum integration time was long enough to get no temporal correlations between frames. The source was selected bright to dominate the readout noise (the limiting noise) effects even in the wings of the signal. Readout noise contribution has been removed from the next results. When this contribution is significant, the removal does not allow a satisfactory accuracy. Consequently, graphs are cut at 2 arcsec. Figure 3 shows the behavior of the standard deviation normalized to the mean computed from the set of psfs with $t=2 \mathrm{~s}$ during the 5 minutes window in function of the radial 


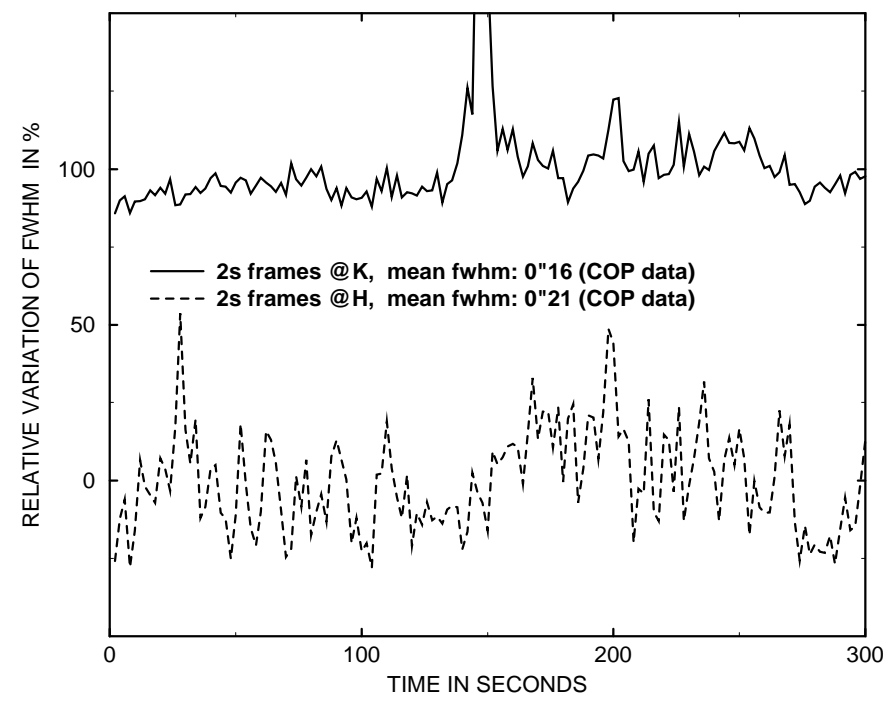

Fig. 2. Same as Fig. 1 but fwhms is plotted instead. Come-on + data

distance to the psf peak. For the purpose of a sequential calibration of the point spread function (see Sect. 4), this term is presumably inversely proportional to the signal to noise ratio. Thus, the signal to noise appears to be quite constant but a bit smaller to the outer halo and near the center.

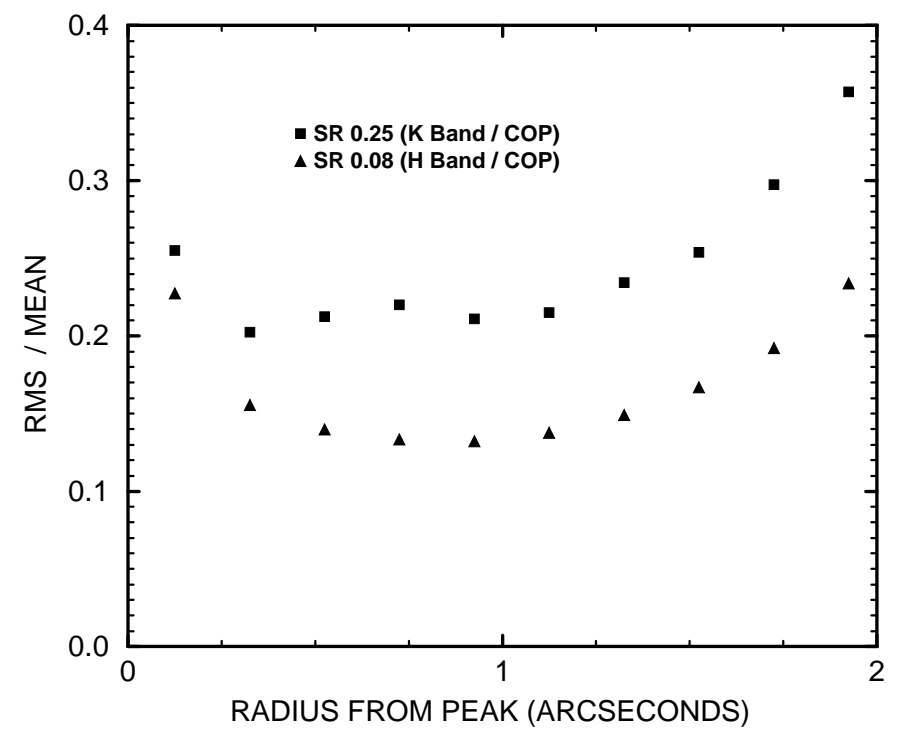

Fig. 3. Standard deviation normalized to the mean value per pixel as a function of its radial distance to the central psf peak from a set of continuous psf frames with $t=2 \mathrm{~s}$ recorded for 5 minutes. Plot is shown for two Strehl ratios: $25 \%$ and $8 \%$ at $\mathrm{KH}$ respectively. The pixel size is 50 mas. Come-on + data

At a given radial distance from the peak, the temporal behavior of the standard deviation for $1<t<20 \mathrm{~s}$ com- puted over the 5 minutes window is well fitted by a power law function as in $t^{-n}$. Figure 4 shows the $\mathrm{n}$ parameter as a function of the radial distance for both $\mathrm{Sr}$. The standard behavior in square root of time corresponds to $n=0.5$ is not found and the signals do not average well with the time, especially near the center. The psf statistics shows atypical behavior which is the reflect of the seeing statistics through the AO system. While the standard deviation normalized to the total energy is as expected lower when the Strehl ratio gets higher, the standard deviation normalized to the mean shown in Fig. 3 increases significantly. Similarly, the temporal behavior for the psf appears to be different with the Strehl ratio as shown by the Fig. 4. This would suggest that the seeing statistics induce amplified effects with $\mathrm{Sr}$ increasing, in the sense that the signal to noise ratio as defined earlier in the residual halo declines. We noted a similar fact for the $r 50$ earlier. These results can be used to assess the consequences for the sequential calibration procedure (see Sect. 4).

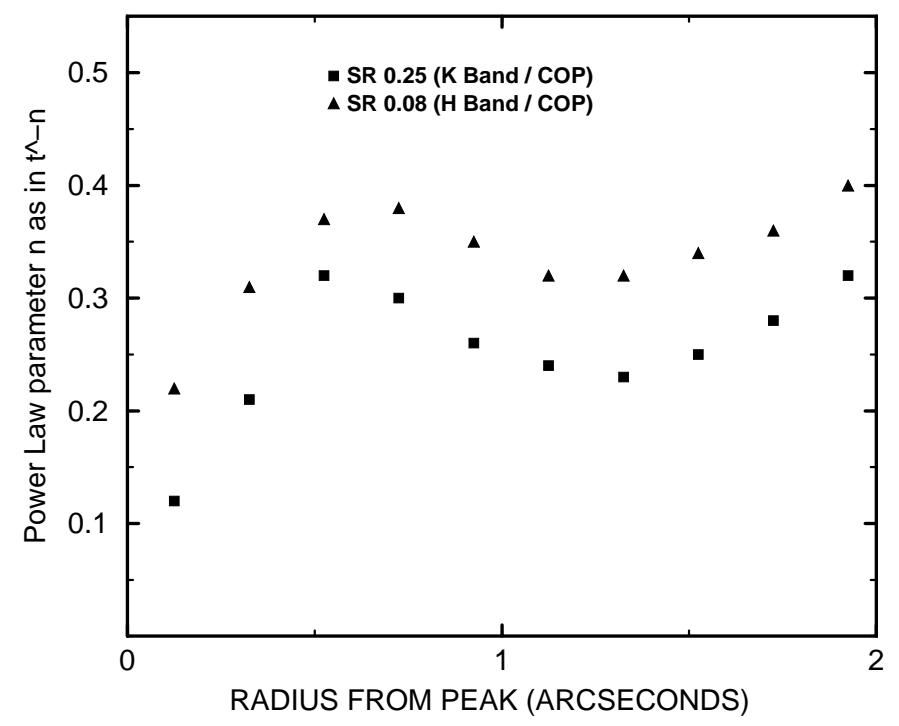

Fig. 4. The standard deviation per pixel (see previous figure) follows a power law as in $t^{-n}$ where $t$ is the integration time of the psf. $n$ is given as a function of the radial distance to the central psf peak. Plot is once again shown for two Strehl ratios: $25 \%$ and $8 \%$ at $K H$ respectively. The pixel size is 50 mas. Come-on + data

The morphology of the on-axis point spread function is now seen for different levels of correction. Figure 5 shows that how the psf halo gets smoother as the integration time increases. The speckle pattern vanishes but some residual features like the secondary mirror spider spikes or the waffle diffraction patterns and also non-identified spatial correlations could be seen on the long-exposure psf. The last ones are not static and could vary. The waffle effect is due to the to the piezo-actuated deformable mirror. 


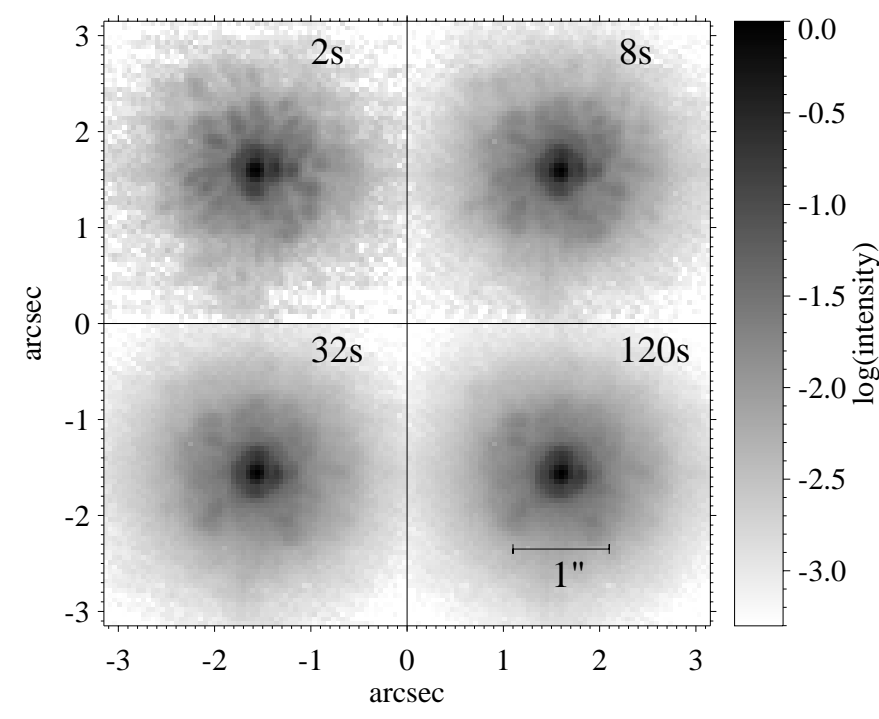

Fig. 5. Psfs integrated over 2, 8, 32 and 120 seconds. The residual speckle pattern is smoothed to a seeing halo. Some known pattern are seen like the waffle diffraction pattern. Sr is about 0.25 in these $K$ images. Come-on + data

Long-exposure psfs from COP do not in general show any major deviation from circular symmetry. In average, the relative deviation is below $20 \%$. However, at a closer range, psfs show some intensity enhancements especially onto the first Airy ring area. These patterns are not fixed and could vary along the night and on short time scale. They do not average out even for long exposure time. Some SOR Gen II psfs have shown a significantly elongated psf. Possible causes are some uncorrected static aberration in the imaging channel, temporal anisoplanatism but in this case, a suspected jitter in the telescope control is probably responsible.

Figures 6 and 7 plot long-exposure psf profiles for different Strehl ratios. These profiles have been obtained by azimuthally averaging the images. Here the profiles are shown normalized to one at the peak. The profiles normalized with the total energy can be obtained by multiplying these previous profiles by their respective Strehl ratio which corresponds to a basic shift for Fig. 7 in logarithmic scale. In linear scale, the profile of the best image available in $K$ with a Strehl ratio of 0.40 compares well with the theoretical diffraction-limited psf. In logarithmic scale, these profiles drops down to $10^{-3}$ pretty close to the source for good corrections. Away, the brightness profiles decrease in parallel lines with a slope of -3 which is consistent with the theoretical profiles of diffraction-limited psfs computed for instruments with obstruction (see Schroeder \& Golimowski 1996). But, the brightness level is higher (1 or 2 orders of magnitude) certainly because of the smallscale imperfection of the optics surfaces.
Linear scale

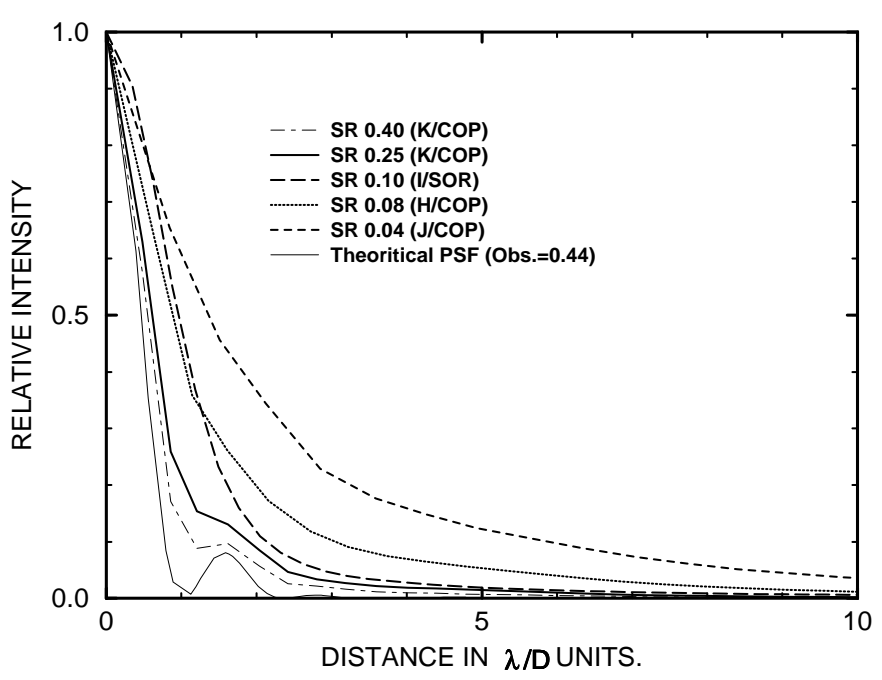

Fig. 6. Profiles of the psfs for different Strehl ratios from COP and SOR Gen II data

Logarithmic scale

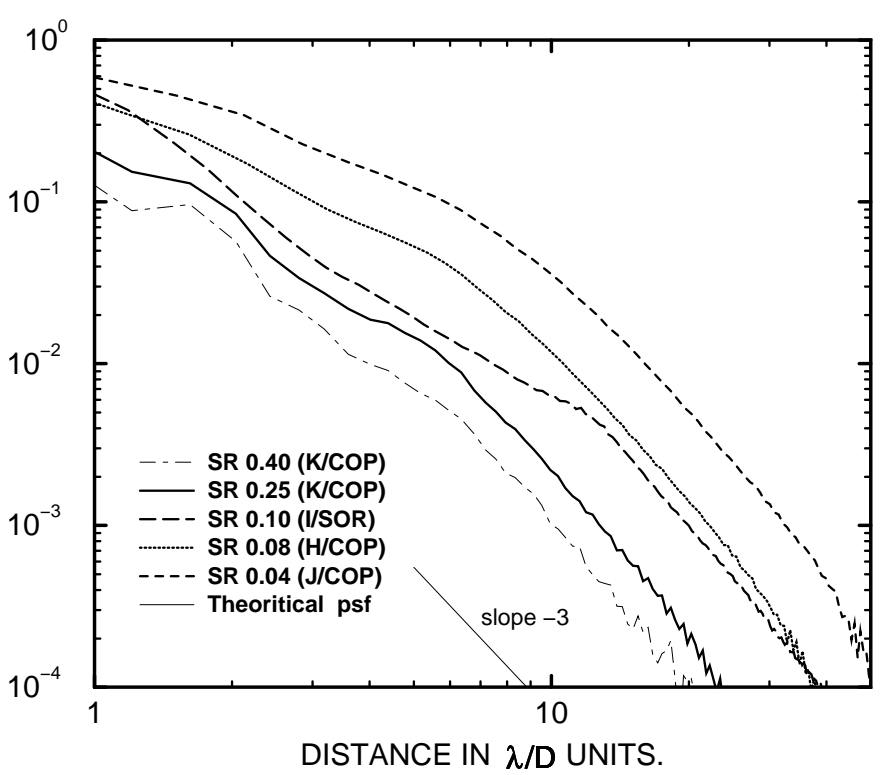

Fig. 7. Same as in Fig. 6 but in logarithmic scale

Psf scalars have been defined in Sect. 1. Full width half maximum and Strehl ratios for various psfs have been located in a diagram in Fig. 8. Psfs are from different observing runs, bands and turbulence conditions. The scale has been normalized to $\lambda / D$. Both parameters appear to be closely related (Tessier et al. 1994; Tessier 1995) as the points clearly gather along a single curve with some scattering. Parameters like the wavelength, the time or the integration time have no measurable effect on the diagram shape. This curve could be also interpreted as an 
AO response curve. More specifically, it is the psf response of the Come-on+ experiment. Incidentally, the curve is pretty well fitted by the basic function defined as:

$\mathrm{Sr}=\left(\beta\left(\frac{\mathrm{fwhm}}{\frac{\lambda}{\mathrm{D}}}-1\right)+1\right)^{-1}$ for $\beta=13$.

As shown by this curve, a fast widening of the psf is observed as soon as the Strehl ratio drops below a value of $10 \%$. This value should be kept in mind as a transition. Images with a Strehl lower than this value will not conserve a diffraction core.

Some psfs from Gen II are overplotted in Fig. 8 and fit again the curve. Most of these psfs were recorded within half an hour where average seeing conditions correspond to $d / r_{0} \approx 1$ and $f_{\mathrm{g}} \approx 40 \mathrm{~Hz}$. The AO bandwidth system was tuned at three different bandwidths to change the correction degree and thus to draw the curve. However, others or future $\mathrm{AO}$ systems may give different responses; due to the limited bandwidth of the two presented systems, the first modes of the turbulence are not totally compensated (see Conan et al. 1995 e.g.); some systems with a higher bandwidth may compensate better the first modes of the turbulence in any conditions and produce low Strehl ratio psf with a sharp core.

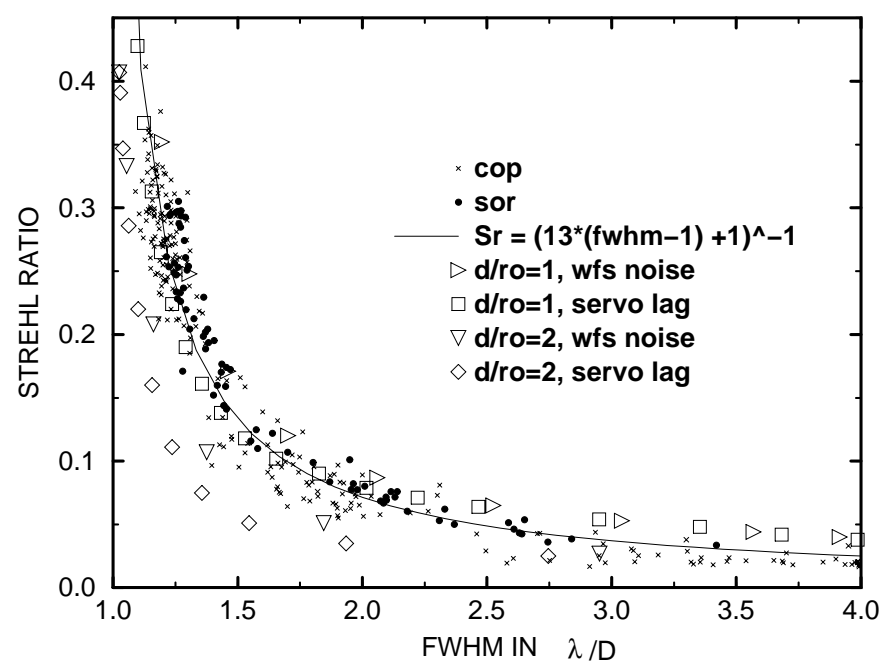

Fig. 8. Position of the psf in a $\mathrm{Sr}-\mathrm{fwhm}$ diagram for the Adaptive Optics system COP in $J H K$ and for SOR Gen II in the $I$ band. Fwhm is rescaled by $\lambda / D$ to show the relationship between $\mathrm{Sr}$ and fwhm. Some theoretical predictions are also plotted. See text

Figure 9 and 10 show the psf location in 50\% energy radius versus fwhm and Sr respectively diagrams. The plots show a scattering larger than in Fig. 8 and appear to be less constrained by the AO system and may vary with the conditions: seeing, wavelength. For example, five SOR psfs corresponding to $d / r_{0} \approx 1$ but very fast turbulence
(Greenwood frequency estimated at $125 \mathrm{~Hz}$ ) are clearly located in a different part of the diagram compared to the other SOR psfs. As a first approximation, $r 50$ is linearly related to $\mathrm{Sr}$ for $\mathrm{Sr}$ between 0.05 and 0.30 . As the $\mathrm{Sr}$ goes below $5 \%, r 50$ increases steeply: some measurements like $(10.4,0.008),(10.7,0.007)$ are out the frame. By contrast with Fig. 8, the two AO systems show significantly different responses (to SOR's advantage). For reference, we give the $r 50$ to fwhm ratio for the theoretical diffractionlimited psf (full correction). This ratio which depends on the obstruction $U$ of the telescope is $\approx 0.5$ for the $1.5 \mathrm{~m}$ SOR telescope $(U=0.07)$, and increases to $\approx 0.75$ for the $3.6 \mathrm{~m}$ ESO telescope $(U=0.44)$; for a pure Gaussian $r 50$ it is equal to 0.5 .

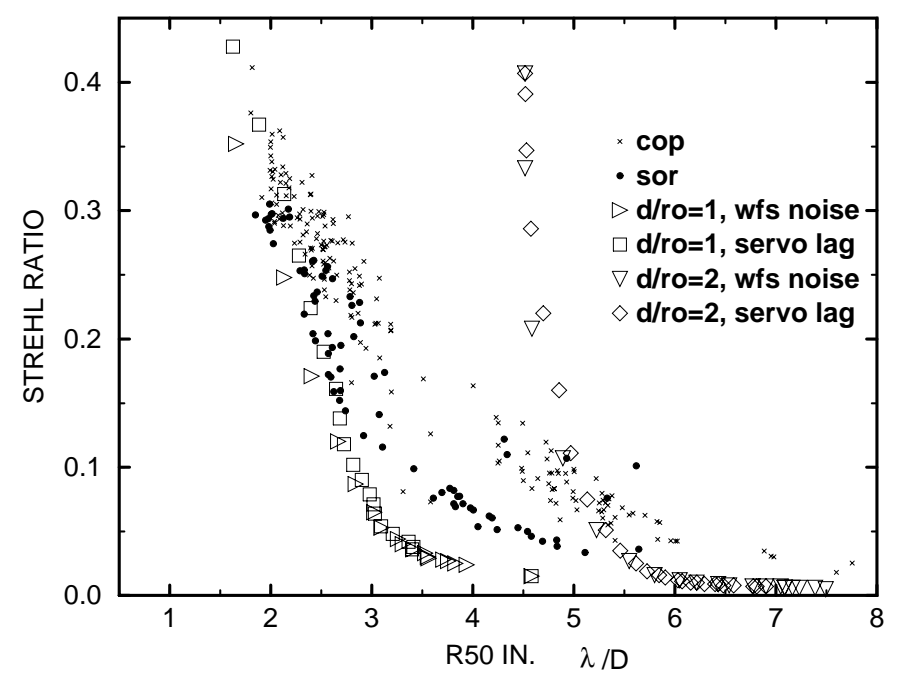

Fig. 9. Same as in Fig. 8 but the psfs are located in a $\mathrm{Sr}-\mathrm{r} 50$ diagram

To recapitulate, the experimental data shown here correspond to bright sources and the following relevant parameters: $D / d=7$ and 16 for COP and Gen II respectively, $d / r_{0}$ between 0.5 and 3 , mostly 1 and 2 in fact (where $d$ is the subaperture width, see Sect. 2). Actually, the high and low Strehl ratios often correspond to $d / r_{0}=1$ and 2 respectively, but fast turbulence has also some effects through the AO bandwidth to Greenwood frequency ratio. Consequently, B. Ellerbroek has kindly generated a set of theoretical optical transfer functions (OTF) with a WFS geometry of $D / d=8$ for the cases $d / r_{0}=1$ and 2 to compare with the COP experimental data. These predictions are based on analytical and covariance calculations in Ellerbroek (1994) which incorporate all the details of the AO system including the modal optimization (Ellerbroek et al. 1994; Gendron \& Léna 1994). Different corrections have been obtained by varying the rms WFS noise (from 0 to about 0.5 waves per subaperture) or the servo lag (translation from 0 to about one subaperture per time constant of the servo in a single turbulent layer 


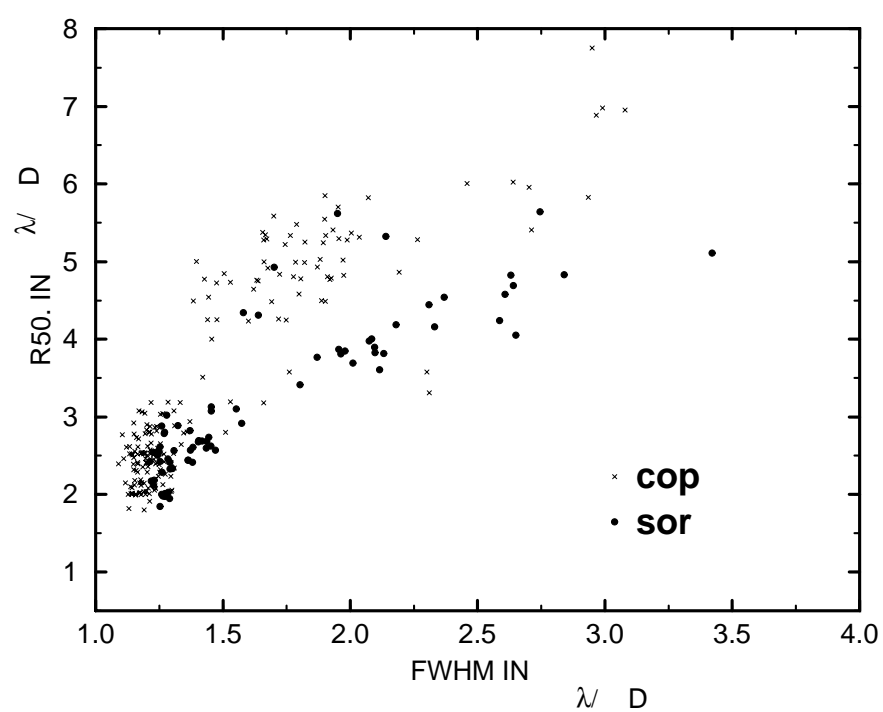

Fig. 10. Same as in Fig. 8 but the psfs are located in a $r 50-$ fwhm diagram

model). Figures 8 and 9 compare the experimental data to the theoretical predictions extracted from the OTFs. The predictions are consistent and satisfactory confirming the close relationship for $\mathrm{Sr}$ versus fwhm while $r 50$ depends a lot on $d / r_{0}$. It is possible that the experimental results are less scattered than the theoretical estimates because the high Strehls were obtained for low values of $d / r_{0}$, and conversely as we have above pointed out.

We now define the scalar fcut as the spatial frequency cutoff at $40 \mathrm{~dB}$ of the power spectrum. fcut is a good indication of the fall-off in the power spectrum (see Sect. 7). However, the spatial frequencies higher than fcut may have not vanished. For example, applying this metric to the pure diffraction-limited psf, fcut is equal to $96 \%$ (in $D / \lambda$ units). Figure 11 shows fcut as a function of the Strehl ratio. The curve shape compares well with Fig. 8. Both systems give the same response as in Fig. 8. In fact, fcut is pretty well linearly related to the fwhm as shown by the plot in Fig. 12 (as expected).

Psf scalars like Sr, fwhm and $r 50$ are in fact a crude description of the psf. Psfs are not perfectly smooth and circular symmetric. Psfs lie in a hyperspace so that it is not realistic to model a psf with a few parameters or with a synthetic function. For a same Sr, profiles and patterns could vary significantly on short time scale and one reminds that from hour to hour, the instrumental transfer function could vary as well; so that the idea to represent this hyperspace by a collection of psfs done once for all science observations is not relevant.

From these results, several conclusions can be drawn:

1. Dynamic range and resolution capability in AO images are confirmed. Diffraction-limit and a high dynamic range are achieved for good $\mathrm{Sr}$.

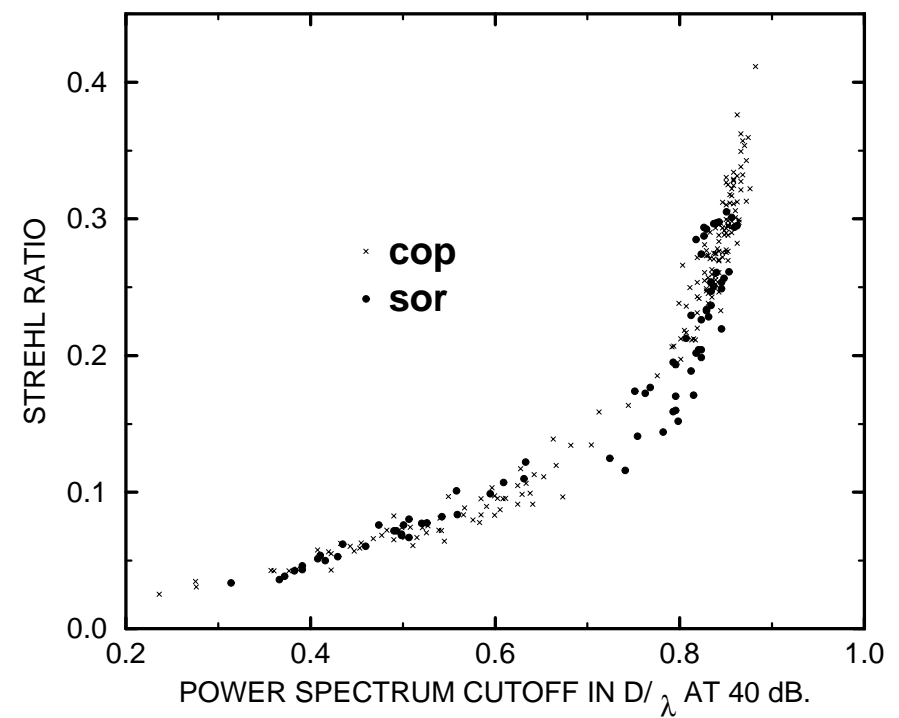

Fig. 11. Same as in Fig. 8 but the psfs are located in a $\mathrm{Sr}-$ fcut diagram. See text

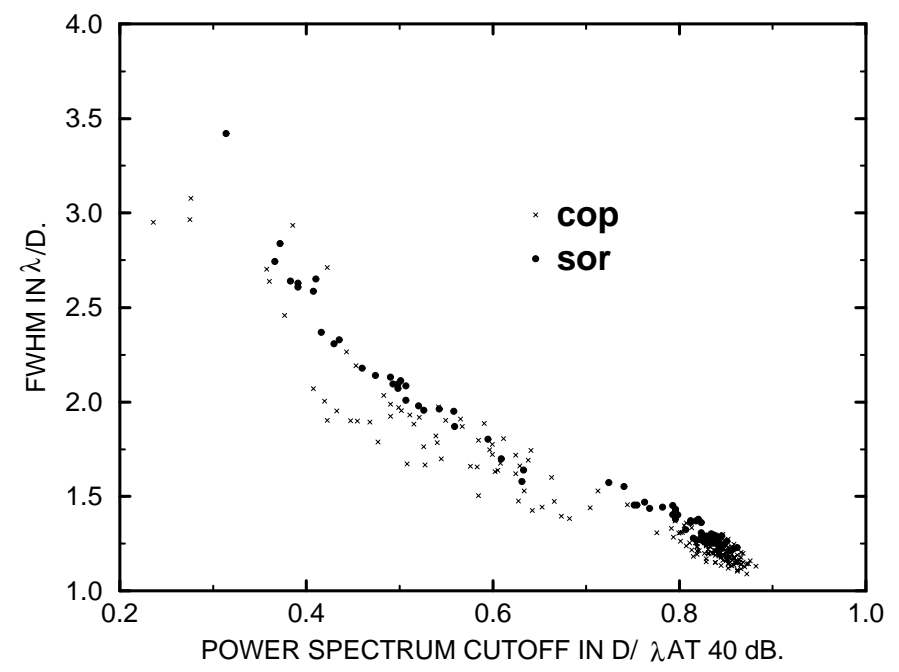

Fig. 12. Same as in Fig. 8 but the psfs are located in a fwhm - fcut diagram. See text

2. The seeing statistics induce continuous change in the correction. In any case, the psfs variations are smoothed with longer exposure times and/or by coadding individual short exposure images.

3. A psf response curve seems to be associated to any given $\mathrm{AO}$ system in the sense that psf scalars are coherently distributed in a diagram. Especially, we note the low scattering for the $\mathrm{Sr}-$ fwhm diagram so that $\mathrm{Sr}$ and fwhm are connected in practice independently of the seeing and the wavelength. These diagrams could be used to direcly infer expected fwhm, $r 50$ from a $\mathrm{Sr}$ estimation for a given AO system and turbulence conditions. We note that for both systems studied here the fwhm degrades dramatically for a Sr smaller than $10 \%$. 
However, the previous diagrams might be AO system dependent.

4. Psf scalars are a crude description of the psf. Indeed, psfs are not perfectly smooth and circular symmetric. Some features could be observed: spike and waffle diffraction patterns appear to be static but enhancement in the first airy ring e.g. seems to be unpredictable.

5. A psf prediction from models or parametrization will not be reliable and only the calibration during the observations can get a fair estimation of the psf. In the partial correction regime, as the psf is sensitive to the seeing variations, any sequential calibration of the psf on a point source will be affected (see Sect. 4).

\section{Calibration of AO images}

The recorded AO image $I$ of a science object is described by the following convolution equation if we ignore anisoplanatism:

$I=O \star \operatorname{psf}(t, \theta)$

where $O$ is the science object intensity distribution. The point spread function knowledge is required to a-posteriori deconvolve AO images (or respectively do model-fitting) and reconstruct the science object intensity distribution. Section 3 has shown that only a calibration during the observations is able to get a good psf estimation. Since seeing statistics are not stationary and the psf is directly affected by the seeing changes, the psfs is a non-stationary variable. Consequently, the psf calibration should be done simultaneously (variable $t$ ) to the science object observations and in the same viewing direction (variable $\theta$ ). Such a technique is not yet available but under testing; Véran et al. (1995) have estimated from simulations this new calibration technique to be relatively accurate to a few per cent. However, this technique has also some drawbacks (Tessier 1995).

The current technique is to calibrate the on-axis psf on a point source (referred to here as the calibrator source) in conditions as similar as possible to the astronomical target source one but sequentially. It is referred to as the sequential calibration technique. In $\mathrm{AO}$, this means to get an equivalent correction on the point source. This will be fulfilled essentially if the WFS noise level is the same. Fortunately, this could be technically done. E.g. the AO system Come-on+ used here is well equipped for this purpose (Beuzit et al. 1994). However, the shape and the color of the source may still induce some differences.

When those precautions are taken, Tessier (1995) has shown on real data that the mismatch in the psf calibration mainly comes from the seeing differences between the observations of the astronomical target and its calibrator point source. The psf charactetistics shown in Sect. 3 demonstrate that the sequential psf calibration is similar to the calibration of the speckle transfer function. Both are sensitive to any temporal variations from the seeing (the so-called seeing effects) or the instrument. We also recall the seeing dependence with the airmass and so the elevation of the source. Some observing procedures are known to improve the calibration in speckle (Perrier 1988), e.g., selecting a calibrator close in the sky, observing both sources within a time as short as possible (a few minutes), and repeating this coming and going between the source and its calibrator a few times if possible. The lattest point is poorly compatible with CCD-like single very long-exposure shot, however, in the infrared, because of the background variations, exposure times are limited in general to five minutes. We have shown that this procedure identically improves the calibration efficiency in adaptive optics (Tessier 1995; Tessier \& Perrier 1996). At last, for a good quality psf, the total integration time must be equal for both science object and its calibrator in order to get a comparable smoothness.

Thus the calibration quality depends on the observational procedure but still depends on the seeing stability which varies with the site and the turbulence conditions. From COP data, I have estimated that the calibration rms error is in relative quite constant over the psf extension and is typically between 5 and $10 \%$ of the signal when one applies the described procedure. On the other hand, if the calibration is not done properly, some bias (stronger halo, wider psf) up to $50 \%$ will show up. To summarize, for a good calibration, it is needed to integrate enough to have a good signal to noise for the halo and to sample well the seeing statistics to overcome the bad averaging capability of the central part of the psf. These points could be related to the peculiar psf statistics shown in Sect. 3. Roddier et al. (1995) have noticed for AO observations carried out at the Canada-France-Hawaii telescope that it was more difficult to calibrate the internal part of the psfs than the external part of the halo.

Blind deconvolution (e.g. Christou 1995) intends to reconstruct the science object intensity distribution with the associated synthetic psf directly from the AO image. However, these algorithms are not able to retrieve the true psf in general. This is not surprising since Sect. 3 has shown how complex the psf is. The problem is illconditioned and needs additional constraints. Thiébaut \& Conan (1995) assume the circular symmetry for the psf, this allows them to reconstruct synthetic psfs. This assumption is not quite satisfactory in the sense that the deviation of the psf to the circular symmetry is currently larger than the uncertainties of the sequential calibration technique. But it could be very useful in the bad case when the psf is unknown, e.g. in the lack of any calibration data.

The sequential calibration technique is not perfect and its accuracy is limited by the seeing statistics to about $5-10 \%$ in relative. However, it is still a very valuable information on how the psf looks like. Blind deconvolution cannot replace this calibration, on the contrary, this information is really needed to constrain near-sighted 
deconvolution algorithms which should be more powerful than standard deconvolution ones (see Sect. 6).

\section{Performance}

The capability to get high-contrast and diffraction-limited images is the strong points of the adaptive optics technique. Performance of $\mathrm{AO}$ is usually described in term of Strehl ratio. As shown in Sect. 3, for Sr higher than 10\%, $\mathrm{AO}$ images allows a resolution up to the diffraction-limit, for lower Strehl, as the psf widens, the fwhm or $r 50$ should be considered as the new resolution (see Fig. 8). As expected, a higher $\mathrm{Sr}$ increases the contrast in the image (see Fig. 7).

However, Tessier (1995) has shown that the psf calibration is the limiting factor to build up high signal to noise (usually done by integrating longer) in the deconvolved images at a given Sr. We will concern the general case where the psf is known from the sequential calibration procedure. To see the consequences of the calibration noise over the performance, I have used typical observations of a bright binary (Tessier et al. 1994). The deconvolution of the AO image using the sequential calibration psf (done as described earlier) have shown residuals stronger than the expected residuals in case of background, photon, readout or flat-fielding noise. These residuals come from the inaccuracy in the calibration process and appear to be the lower limit of the noise. Figure 13 shows the limiting magnitude for an unknown companion detection in presence of these residuals levels. The Strehl ratio greatly improves the detection capability. For good Sr, the dynamic range of the detection regularly increases when going away from the central source from $10^{2}$ at 0.5 arcsec up to $10^{4}$ at 2 arcsec. An extended source could be defined as a source much larger than the $50 \%$ energy radius of the psf. Sensitivity curves for such sources are shown in Fig. 14 for a pixel of 50 mas. Binning pixels will gain in sensitivity as the binning factor. For the $J$ curves ( $\mathrm{Sr}$ of $4 \%$ ), the sensitivity is probably underestimated since we know that the psf calibration was quite poor in this case.

The visibility defined as the ratio of the AO image Fourier spectrum to the calibration psf one is also concerned by the calibration noise. An unresolved source will show some deviation to the flat visibility: e.g. in the visibility in modulus, a few per cent for good calibration and bias larger than $50 \%$ is usual in case of miscalibration.

Science observations have been carried out for a few years and yielded some results which set the current abilities in AO. Resolution up to the diffraction limit is possible on AO images. See e.g. the binary with a separation equal to the diffraction limit in Tessier et al. (1994) and Brandner et al. (1995). However, unpredictable feature on the first Airy ring may limit the detection level in contrast. Léna (1994) has collected current scientific publications from COP data. First, the observation of the R 136 cluster has revealed stars in the field (13 arcsec) as faint

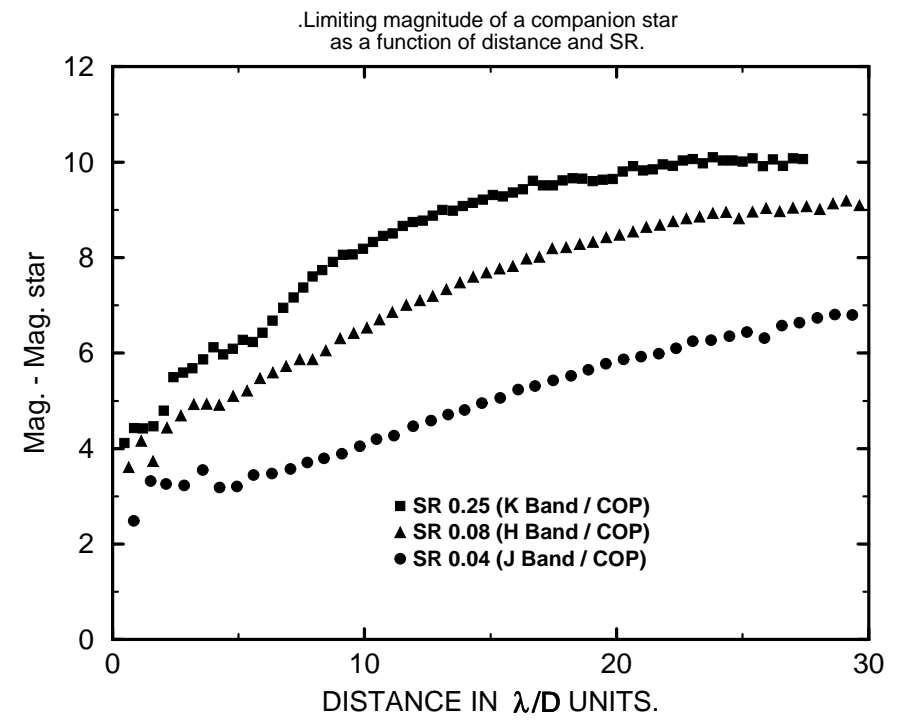

Fig. 13. COP sensitivity curve for the detection of a companion in magnitude difference to the main component as a function of the separation for different Strehl ratios. Plot is rescaled by $\lambda / D$. A pixel width of 50 mas is assumed

as a magnitude difference of 9 relatively to the brightest star in the field (see also Brandl et al. 1995); secondly, a faint companion in $K$ with a flux ratio of $10^{4}$ at 4 arcsec have been detected around the object HR 4796, and the detection of the disk around $\beta$ Pictoris (Lagrange et al. 1996) at 2 arcsec. All these experimental results are consistent with the detectivity curves shown previously. From close binaries observations (a few $\lambda / D$ ) I have personally processed, the relative accuracy is a few milli-arcseconds for astrometry, a few per cent for relative photometry.

\section{Deconvolution of AO images}

One can question the validity of standard deconvolution in AO. Standard deconvolution algorithms (Lucy, MEM, CLEAN) assume an exact psf. Though it is not the case for AO images, these algorithms could work. Charter (1992) considers that the correct probabilistic approach should be to integrate over uncertainties in the psf. But he says that choosing the psf at its modal value is a good approximation if the psf distribution probability is sharply peaked. It should be the case when the calibration is done carefully. However, it is quite difficult to estimate in the deconvolved image the error level and consequently the features which may come from the psf miscalibration. For example, the strength of the MEMSYS-4 algorithm (Copyright (C)MEDC 1990, Gull 1989), compared to the previous ones is to provide a stopping criterion and a final error map. However, this map does not take in account the psf uncertainties and error could be underestimated (Charter 1992). 


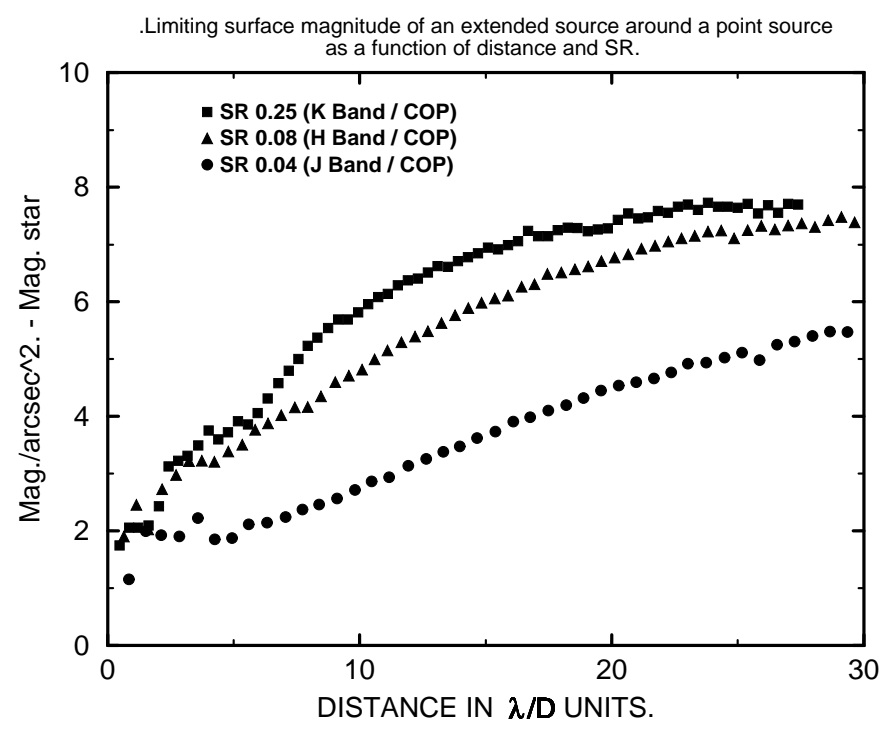

Fig. 14. COP sensitivity curve for the detection of an extended structure around a point source in magnitude per arc-second ${ }^{2}$ relative to the central source as a function of the radial distance for different Strehl ratios. Plot is rescaled by $\lambda / D$. A pixel width of 50 mas is assumed

Since AO images deconvolved with standard algorithms are limited by the uncertainties in the psf knowledge but not by the signal to noise in the AO image, it seems interesting to use blind deconvolution algorithm. Looking for a solution to Eq. (2) with an unknown object and a psf lying in a hyperspace looks challenging. The main problem is to well-condition the problem in order to get a unique solution. Indeed these algorithms are not satisfactory with no further constraint on the psf. Christou \& Drummond (1995) have proposed the model-fitting blind deconvolution which assumes an a-priori knowledge of the object and/or the psf. From HST images of crowded star clusters with some isolated point sources in the isoplanetic field (on the edges for example), White (1994) enforces the presence of point sources in the restored object image to constrain the blind deconvolution process. We now address the general case of any object. Christou (1995) has proposed to work on several AO images with different psfs but based on the same object and has obtained some promising results. Reducing the dimension of the hyperspace is certainly a solution. Circular symmetry assumption for the psf yields interesting results (Thiébaut $\&$ Conan 1995). But it is possible to constrain the psf with the calibration psf. So, I have defined a loose constraint defined as to minimize the psf distance from the calibration psf. In a blind deconvolution algorithm (see Thiébaut \& Conan 1995 e.g.), this constraint is seen as an additional term in the error metric. This term is given by:

$\epsilon_{\mathrm{c}}=\left\|\mathrm{psf}-\mathrm{psf}_{\mathrm{c}}\right\|^{2}$ where $\operatorname{psf}_{c}$ is the calibration psf. Because of this loose constraint, the blind deconvolution algorithm could be renamed near-sighted deconvolution. The overall error metric could be redefined as follows:

$$
\epsilon=\frac{1}{1+\alpha} \epsilon_{\mathrm{d}}+\frac{\alpha}{1+\alpha} \epsilon_{\mathrm{c}}
$$

where $\alpha$ is called a hyper-parameter and $\epsilon_{\mathrm{d}}$ is the usual error metric in blind deconvolution. $\alpha=0$ corresponds to blind deconvolution and $\alpha=\infty$ corresponds to standard deconvolution.

I propose to control the blind deconvolution process by decreasing $\alpha$ from a large value to zero. For example, first step could be to start with $\alpha=100$, as initial guesses the AO image and a wide Gaussian for the deconvolved object image and the psf respectively. At each step, one decreases $\alpha$ by a factor 10 and takes the previous step solutions as the initial guesses. One stops the process when there is no more significant gain in the error $\epsilon_{\mathrm{d}}$ or there is obvious divergence for the synthetic psf.

This new constraint has been easily incorporated in the error metric of the deconvolution code written by Thiébaut \& Conan (1995). Data from a 0.126 arcsec binary observations (Tessier et al. 1994; Brandner et al. $1995)$ taken at $J$ with a Sr of about $3 \%$ have been used to test this method. The calibration psf available was taken 45 minutes after the binary observations. So, we are not sure about its reliability. This is a difficult test because the overlapping psfs and the psf shape complexity make tricky for identifying the binary. Figure 15 shows that this process helps to resolve the binary better. For low $\alpha$, as the psf constraint is loosed, some noise appears in the halo of the synthetic psf. That means that the problem starts to be ill-conditioned again, probably because the psf halo size and dynamic range are too large, so there are too many degrees of freedom. One better stops the process. Incidently, in order to overcome this problem, one might use the wavelet transform for a multiple resolution analysis (see e.g. Starck \& Murtagh 1994). Using the multiscale decomposition for the psfs, Eq. (3) can be expanded as:

$\epsilon_{\mathrm{c}}=\sum_{i} \alpha_{i}\left\|\left\{\mathrm{psf}-\mathrm{psf}_{\mathrm{c}}\right\}^{i}\right\|^{2}$

where $\{f\}^{i}$ denotes the wavelet plane for $f$ at scale $i$, $\alpha_{i}$ is the weight at scale $i$. Appropriate set of $\alpha_{i}$ with possibly use of thresholding may help to solve the previous problem.

This binary was revealed from HST observations in the visible (Bernacca et al. 1993). The separation and the position angle (PA) were measured to 0.126 arcsec \pm 0.007 arcsec and $63.4 \mathrm{deg} \pm 1.0 \mathrm{deg}$ respectively. Table 3 lists the binary parameters extracted from the AO deconvolved images in $J$, it shows a $20 \%$ discrepancy for the photometry between the near-sighted deconvolution and 


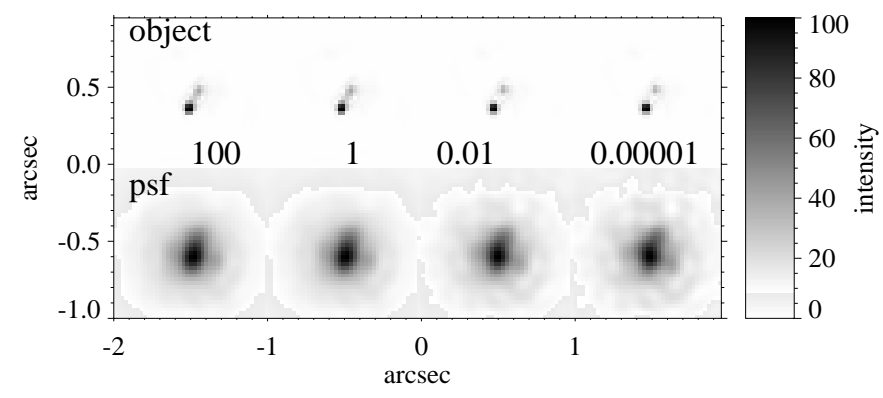

Fig. 15. Results of blind deconvolution controlled with the calibration psf for decreasing $\alpha$. See text

the standard deconvolution methods. We have to wait additional observations in $J$ for confirmation but the nearsighted deconvolution process might have retrieved the relevant information.

All these results are still preliminary but the nearsighted technique certainly deserves further investigation.

Table 3. Binary parameters from the deconvolved images. Done with the task phot from daophot

\begin{tabular}{|l|l|l|l|}
\hline $\begin{array}{l}\text { Deconvolution } \\
\text { method }\end{array}$ & $\begin{array}{l}\text { magnitude } \\
\text { difference }\end{array}$ & $\begin{array}{l}\text { PA } \\
\text { (degree) }\end{array}$ & $\begin{array}{l}\text { separation } \\
\text { (arcsec) }\end{array}$ \\
\hline CLEAN & 0.81 & 59.1 & 0.121 \\
Lucy & 0.75 & 60.1 & 0.133 \\
MEMSYS-4 & 0.74 & 61.4 & 0.139 \\
Near-sighted & 0.96 & 61.9 & 0.139 \\
\hline
\end{tabular}

\section{Speckle with adaptives optics}

Rigaut et al. have observed (1991) an enhancement of the speckle transfer function by up to a factor 7 using the AO prototype Come-on. From AO calculations and simulations in the partial correction regime, Roggemann \& Matson (1992) and Conan et al. (1993) showed that the speckle transfer function with AO yields a significant gain in signal and signal to noise ratio compare to the ones obtained without AO. From AO simulations, Roggemann \& Matson (1992) have also shown that bispectral analysis yields a significant gain for the partially compensated case.

Tessier \& Perrier (1996) have carried out some Comeon + observations in speckle mode. A critical value for the Strehl ratio is observed for long exposure AO images. In the images with a Sr lower than about $5 \%$, the diffraction-limited coherent peak is lost as the optical transfer function does not show anymore high spatial frequencies (dashed line in Fig. 16). The use of short exposure times allows to recover the high spatial frequencies part in the modulation transfer function (MTF) shown in solid line in Fig. 16. In our case, the $250 \mathrm{~ms}$ exposure time in $J$ was quite long in comparison to the typical speckle coherence time estimated from the wavefront sensor data. This suggests that a speckle analysis improves the high frequencies even with exposure times larger than the speckle coherence time. By contrast, in case of good $\mathrm{Sr}(>10 \%)$, this mode does not yield so much gain and is not relevant.

The behavior of the signal to noise depends more on the quality of the MTF calibration possibly achievable. We have completed the observation of a close binary $(0.2$ arcsec $)$ using this technique. The data have been reduced with the IRAF package c128 (see Appendix B; Tessier et al. 1994). From the rms residual of the reconstructed complex visibility, we can conclude that the phase is much less sensitive to seeing effects than the modulus is. The rms residual for the phase is about 0.03 radians from 500 frames. The rms residual for the visibility in modulus is $3 \%$ in the high spatial frequency range but some seeing effects affect up to $20 \%$ the low spatial frequency region $\left(r_{0} / \lambda\right)$.

To conclude, the field of application of speckle with AO applies essentially to low corrections (a few per cent). This occurs when, e.g., going short-wards in wavelength for given conditions. We should be able to observe fainter sources with a higher resolution than in speckle without AO because of the gain in signal and the possibility to use longer exposure times.

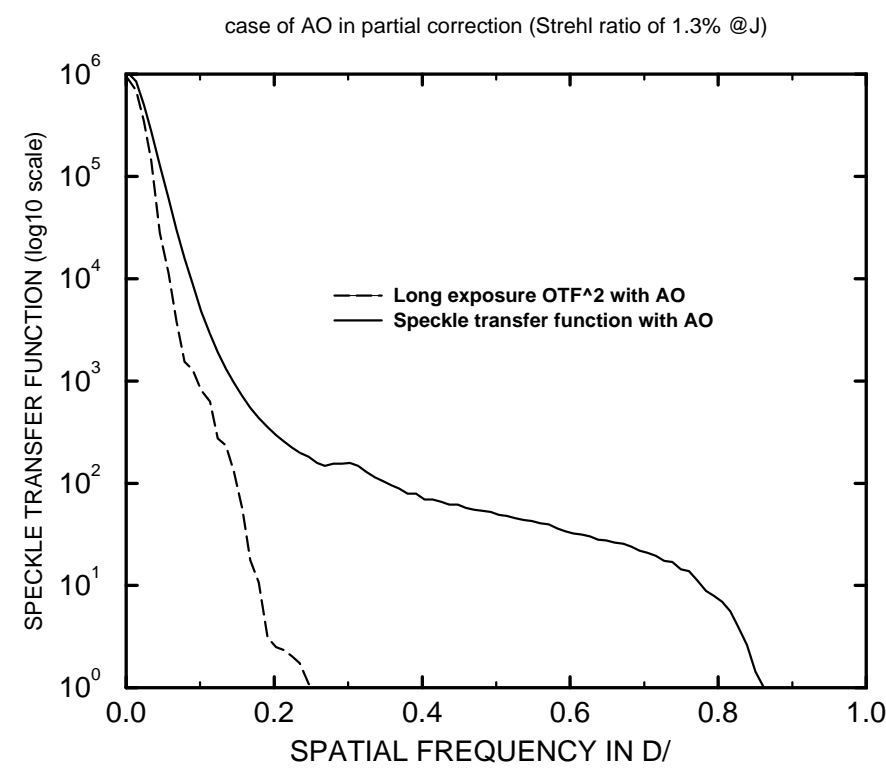

Fig. 16. Speckle transfer function of short exposures AO images (exposure time is $0.25 \mathrm{~s}$ in $J$ band) under poor correction compared to the square optical transfer function of the long-exposure AO image. From COP data

Speckle imaging is a post-processing technique based on sets of short exposure images. When a set of $\mathrm{AO}$ 
images is available from the science object and its calibration psf, it may be useful to test some techniques drawn from the speckle experience before applying the deconvolution. First, it could be necessary to recenter the frames on their centroid before averaging the sets. Roddier et al. (1995) have used the median operator in place of the mean on their sets of images with some success. Other operators could be tested. The rebinning technique initially used in speckle (Perrier 1988) may be applied here and should improve the sequential calibration procedure accuracy by minimizing the "correction" effects (due to the seeing fluctuations e.g.). It consists in binning object and psf AO images with the same level of correction. This technique requires the object-independent estimation of the Strehl ratio in the AO images. Incidentally, the AO system ADONIS has been providing this feature recently: the Strehl ratio is estimated from the WFS data (E. Gendron, private communication), so that this technique could be now tested. Some best estimator should yield the final AO image and its calibration psf from these bins. The selection technique is another possibility which aims to increase the sharpness in the images by discarding the bad images in the set (Tessier et al. 1994). However to get a good calibration psf, the same operations must be carried out on the set of the psf images. In fact, this technique is equivalent to the rebinning technique where the bad bins are rejected.

\section{Conclusion}

We have processed NGS AO data with a Sr ranging from a few per cent to 0.4 which are typical values achieved by operational AO systems under average seeing conditions and corresponds to the partial correction regime. Psf calibration actually sets the limits in extracting information from AO deconvolved images. Despite this limitation, accuracies in the AO images of a few mas in relative astrometry and a few per cent in relative photometry, with a dynamic range up to $10^{4}$ and a diffraction-limited resolution are achievable on the current systems. These performances should be improving in a near future thanks to better calibration/deconvolution processes or if higher Strehl ratios are systematically achieved. Among the solutions, we have seen a promising near-sighted deconvolution which might be a way to fully use the information contained in the $\mathrm{AO}$ images.

\section{A. Strehl ratio computation}

The performance of an AO system is usually described in term of Strehl ratio. The Strehl ratio is the ratio of the psf maximum intensity to the theoretical diffraction-limited psf one; the latter is the Airy pattern in the case of a clear circular aperture telescope of diameter D. We present the steps to compute an unbiased estimation of the Strehl ratio from any $\mathrm{AO}$ image of a point source provided that the Nyquist criterion is fulfilled for the effective spatial frequency cutoff.

For a theoretical diffraction-limited psf centered, sampled by a Shah function, and with energy normalized to one, the discrete peak value $M_{0}$ at zero position is given by the formula:

$M_{0}=\frac{\pi}{16} \gamma^{-2}\left(1-U^{2}\right)$

where $\gamma$ is the ratio of the Shah function sampling frequency to the Nyquist frequency $(2 D / \lambda)$, and $U$ is the linear central obstruction of the telescope. Let be an $\mathrm{AO}$ image sampled by a $N \times N$ array of detectors. In order to compare with the previous psf, we first normalize the image energy to 1.0 and defilter from the pixel function (for a square pixel with a filling factor $g$, one divides the discrete Fourier transform of the image by the function $\left.\operatorname{sinc}\left(g^{1 / 2} \nu_{x} / N\right) \operatorname{sinc}\left(g^{1 / 2} \nu_{y} / N\right)\right)$. The defiltering operation is not needed in case of smooth peak or oversampled images $(\gamma \geq 1.5)$. The peak position in the image is then located from a spline function interpolation. The image is shifted to this position via the Fourier transform and not via a spline interpolation. Using Eq. (A1), the Strehl ratio of the AO image is thus given by:

$\mathrm{Sr}=M \frac{16}{\pi} \gamma^{2} \frac{1}{1-U^{2}}$

where $M$ is the peak value in the shifted image. Of course, if we use a non point source image, the Sr value will be under-estimated all the more as the source is resolved. Assuming data without noise, the error source in this technique comes from the position estimated from the spline interpolation. For synthetic psfs which have been shifted by fractional pixel values in $x$ and $y$, the maximum position error is always $\leq 0.1$ pixels which yields to a marginal relative error for the Strehl ratio $\leq 10^{-2}$.

\section{B. Image processing software}

c128 is an IRAF package for processing imaging data from either adaptive optics or speckle instruments preferably in the infrared since the photon noise contribution is not taken into account. The main strength of c128 is to handle cubes of images. It has been extensively used to produce the results shown in this document. c128 is a standard IRAF package with an online help and is available on anonymous-ftp from the hplyot.obspm.fr internet-site in the iraf_hra directory. c128 is provided for the convenience of all users with no warranty of accuracy or usability.

Acknowledgements. This work has been funded by the Human Capital \& Mobility programme for the European Union network on high resolution imaging. This network gathers various European institutes involved in high angular resolution techniques for astronomy. I thank J. Bouvier, C. Perrier and J. Christou, B. Ellerbroek for providing COP data and SOR Gen II data respectively. I thank E. Thiébaut and W. Brandner 
for providing the blind deconvolution algorithm BDEC and an IDL script respectively. The theoretical AO OTFs were generated by B. Ellerbroek to whom I am thankful. I thank B. Ellerbroek for critically reading the manuscript and for fruitful discussions. I thank C. Jenkins for carefully reading the manuscript and making useful comments. Special thanks to Brent Ellerbroek and the SOR team for the hospitality and support shown to me while an observer at the Starfire Optical Range. I wish to thank R. Foy and M. Redfern for their support.

\section{References}

Bernacca P., Lattanzi M., Bucciarelli B., et al., A\&A 278, L47

Beuzit J.-L., Hubin N., Gendron E., et al., 1994, in Adaptive Optics in Astronomy, Ealey M.A., Merkle F. (eds.) Proc. SPIE 2201, 955

Brandl B., Sams B., Bertoldi F., 1996, ApJ 466, 254

Brandner W., Bouvier J., Grebel E.K., et al., 1995, A\&A 298, 818

Charter M.K., 1992, in Maximum Entropy and Bayesian Methods, Mohammad-Djafari A., Demoment G. (eds.). Kluwer, Dordrecht

Christou J., 1995, in Adaptive Optical Systems and Applications, Robert K. Tyson, Robert Q. Fugate (eds.) Proc. SPIE 2534, 226

Christou J., Drummond J., 1995, in ESO-OSA Topical Meeting on Adaptive Optics, Cullum M. (ed.). Garching, Germany, Oct. 1995 , p. 323

Conan J.-M., Madec P.-Y., Rousset G., 1993, Proceedings of ICO-16 Satellite Conference on Active and Adaptive Optics, Merkle F. (ed.). ESO, Garching bei Munchen, Germany, p. 181

Conan J.-M., Madec P.-Y., Rousset G., 1995, J. Opt. Soc. Am. A 12, 1559

Ellerbroek B., 1994, J. Opt. Soc. Am. A 11, 783

Ellerbroek B., Van Loan C., Pitsianis N., et al., 1994, J. Opt. Soc. Am. A 11, 2871

Fugate R., Ellerbroek B., Higgins C., et al., 1994, J. Opt. Soc. Am. A 11, 310
Gendron E., Léna P., 1994, A\&A 291, 337

Gull S.F., 1989, in Maximum Entropy and Bayesian Methods, Skilling J. (ed.). Kluwer, Dordretch, p. 53

Johnston D., Brown II J., Christou J., et al., 1996, in OSA Topical Meeting on Adaptive Optics, Maui, U.S.A., Jul. 1996, Technical Digest 13, p. 158

Lagrange A.-M., et al., 1996 (in preparation)

Léna P., 1994, in Adaptive Optics in Astronomy, Ealey M.A., Merkle F. (ed.) Proc. SPIE 2201, 1099

Perrier C., 1988, in Diffraction-Limited Imaging with Very Large Telescopes, NATO ASI Ser. C 274, 99, Alloin D.M. and Mariotti J.-M. (eds.). Kluwer, Dordrecht

Rigaut F., Kern P., Léna P., et al., 1991, A\&A 250, 280

Roddier F., 1994, in Adaptive Optics for Astronomy, NATO ASI Ser. C 423, 12, Alloin D.M. and Mariotti J.-M. (eds.) Kluwer, Dordrecht, p. 89

Roddier F., Roddier C., Graves J.E., et al., 1995, ApJ 443, 249

Roggemann M.C., Matson C.L., 1992, J. Opt. Soc. Am. A 9, 1525

Schroeder D., Golimowski D., 1996, PASP 108, 510

Starck J.-L., Murtagh F., 1994, A\&A 288, 342

Tessier E., Bouvier J., Lacombe F., 1994, A\&A 283, 827

Tessier E., Bouvier J., Beuzit J.-L., et al., 1994, The Messenger 78,35

Tessier E., 1995, in Adaptive Optical Systems and Applications, Robert K. Tyson, Robert Q. Fugate (eds.) Proc. SPIE 2534, 178

Tessier E., Perrier C., 1996, in OSA Topical Meeting on Adaptive Optics, Maui, U.S.A., Jul. 1996, Technical Digest, p. 13

Thiébaut E., Conan J.-M., 1995, J. Opt. Soc. Am. A 12, 485

Véran J.-P., Rigaut F., Maître H., 1995, in ESO-OSA Topical Meeting on Adaptive Optics, M. Cullum (ed.). Garching, Germany, Oct. 1995, p. 497

Wilson R.W., Jenkins C.R., 1996, MNRAS 278, 39

White R., 1994, in the Restoration of HST Images and Spectra II, Space Telescope Science Institute, 1994, Hanisch R.J. and White R.L. (eds.) p. 198 